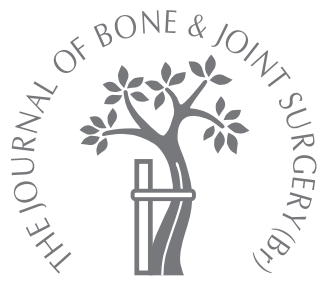
A. Gupta,
J. Meswania,
R. Pollock,
S. R. Cannon,
T. W. R. Briggs,
S. Taylor,
G. Blunn

From The Royal

National

Orthopaedic

Hospital NHS Trust,

Middlesex, England

A. Gupta, MBBS, MS(Orth) MRCS, Specialist Registrar

R. Pollock, FRCS(Tr \& Orth),

Consultant Orthopaedic

Surgeon

S. R. Cannon, FRCS,

MCh(Orth), Consultant

Orthopaedic Surgeon

T. W. R. Briggs, FRCS,

MCh(Orth), Consultant

Orthopaedic Surgeon

Department of Orthopaedic

Oncology

The Royal National

Orthopaedic Hospital NHS

Trust, Stanmore, Middlesex

HA7 4LP, UK

‥ J. Meswania, BSc, Senior

Research Fellow

S. Taylor, PhD, Lecturer

- G. Blunn, PhD, Professor

Department of Biomedical

Engineering

Institute of Orthopaedics and

Musculoskeletal Sciences,

University College London,

Gower Street, London WC1E

6BT, UK.

Correspondence should be sent

to Mr A. Gupta; e-mail:

ajaygupta@hotmail.co.uk

(C)2006 British Editorial Society of Bone and Joint Surgery doi:10.1302/0301-620X.88B5.

$17098 \$ 2.00$

$J$ Bone Joint Surg [Br]

2006;88-B:649-54.

Received 17 August 2005

Accepted after revision

6 January 2006

\title{
Non-invasive distal femoral expandable endoprosthesis for limb-salvage surgery in paediatric tumours
}

\begin{abstract}
We report our early experience with the use of a non-invasive distal femoral expandable endoprosthesis in seven skeletally immature patients with osteosarcoma of the distal femur. The patients had a mean age of 12.1 years (9 to 15) at the time of surgery. The prosthesis was lengthened at appropriate intervals in outpatient clinics, without anaesthesia, using the principle of electromagnetic induction. The patients were functionally evaluated using the Musculoskeletal Tumour Society scoring system. The mean follow-up was $\mathbf{2 0 . 2}$ months (14 to $\mathbf{3 0}$ ). The prostheses were lengthened by a mean of $25 \mathrm{~mm}$ (4.25 to 55) and maintained a mean knee flexion of $110^{\circ}\left(100^{\circ}\right.$ to $\left.120^{\circ}\right)$. The mean Musculoskeletal Tumour Society score was 68\% (11 to 29). Complications developed in two patients; one developed a flexion deformity of $25^{\circ}$ at the knee joint, which was subsequently overcome and one died of disseminated disease. The early results from patients treated with this device have been encouraging. The implant avoids multiple surgical procedures, general anaesthesia and assists in maintaining leg-length equality.
\end{abstract}

The distal end of the femur is the most common site for primary malignant tumours of bone in children, with osteosarcoma being the most common. Over the last three decades, increased knowledge about the disease process and advances in imaging modalities, adjuvant chemotherapy and surgical techniques, have allowed orthopaedic oncology to avoid amputation in favour of limb salvage for patients with sarcomas. $^{1-3}$

Limb-salvage operations have shown a local recurrence rate of between $5 \%$ and $10 \%$, comparable with that achieved by amputation. ${ }^{4-7}$ Today, $90 \%$ of patients with a sarcoma of the extremity are candidates for limb preservation and the chances of long-term survival (ten years) should approach $70 \%$ to $80 \% .^{8}$ However, encroachment of the tumour on major neurovascular structures, pathological fractures with contamination of local tissue and local recurrence after the salvage operations, may still require amputation. ${ }^{9}$

Limb-salvage operations with fixed-length endoprostheses in skeletally immature patients lead to limb-length inequalities at the end of the growth phase. ${ }^{10}$ Implant development has seen the introduction of extendable endoprosthetic replacements as an established and safe alternative. ${ }^{11-17}$ The extendable prosthetic module compensates for discrepancies in leg length which occur in growing patients with a fixed- length implant. These prostheses, which are extended at operation, are good functionally and psychologically, although each open procedure is accompanied by the risk of ankylosis, nerve damage and infection and in some cases amputation. ${ }^{11,16}$ Non-invasive designs, which avoid these complications have been described. ${ }^{18-21}$

We have developed the 'non-invasive distal femoral expandable endoprosthesis - the juvenile tumour system', a prosthesis which can be lengthened non-invasively via electromagnetic induction. We have examined the early clinical results in patients with this type of prosthesis.

\section{Patients and Methods}

The non-invasive distal femoral expandable endoprosthesis has a femoral and a tibial component articulating at the knee joint, forming a total knee replacement. The shaft of the femoral component, shown in Figure 1, is constructed in two segments which slide longitudinally during the lengthening process. The stemmed proximal end of the femoral shaft is cemented into the remaining segment of the femur, while the distal end of the femoral component articulates with the tibial component. The prosthesis is implanted like any normal massive replacement. Embedded within the telescoping shaft are a magnetic disc, a gearbox and a power screw, which enable the shaft of 


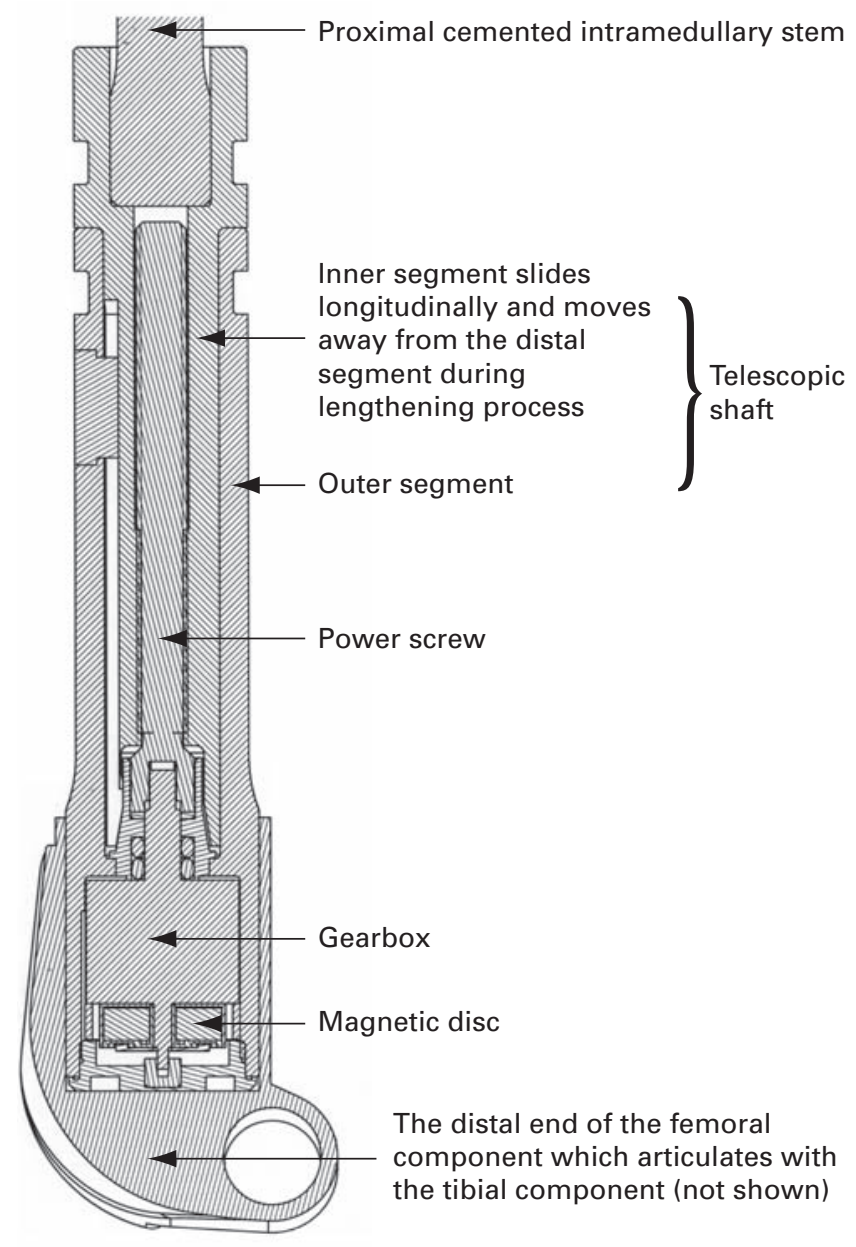

Fig. 1

Section through the femoral component of the non-invasive distal femoral expandable endoprosthesis.

the prosthesis to be lengthened. The magnetic disc is made from rare earth magnetic material and is connected to the input shaft of the gearbox (Fig. 1). The gearbox has an epicyclic gear train with 13 061:1 reduction, an overall diameter of $21.5 \mathrm{~mm}$ and length of $18.5 \mathrm{~mm}$. The output shaft of the gearbox is connected to a threaded screw connecting with the inner telescopic segment. As the screw rotates, it drives the inner and outer segments of the telescopic shaft apart, thereby extending it. Under normal use, the gearbox is static. An external drive unit with large electric coils can induce a rotating magnetic field at a speed of 3000 RPM when electric current is passed through the coils. When the prosthesis is placed at the centre of the rotating electromagnetic field, the poles of the magnet within the implant are captured, causing it to rotate in synchrony (Fig. 2). Because the external field rotates at a fixed speed, implant extension is achieved at a rate of $0.23 \mathrm{~mm}$ per minute, equating to approximately $1 \mathrm{~mm}$ of lengthening every four minutes.

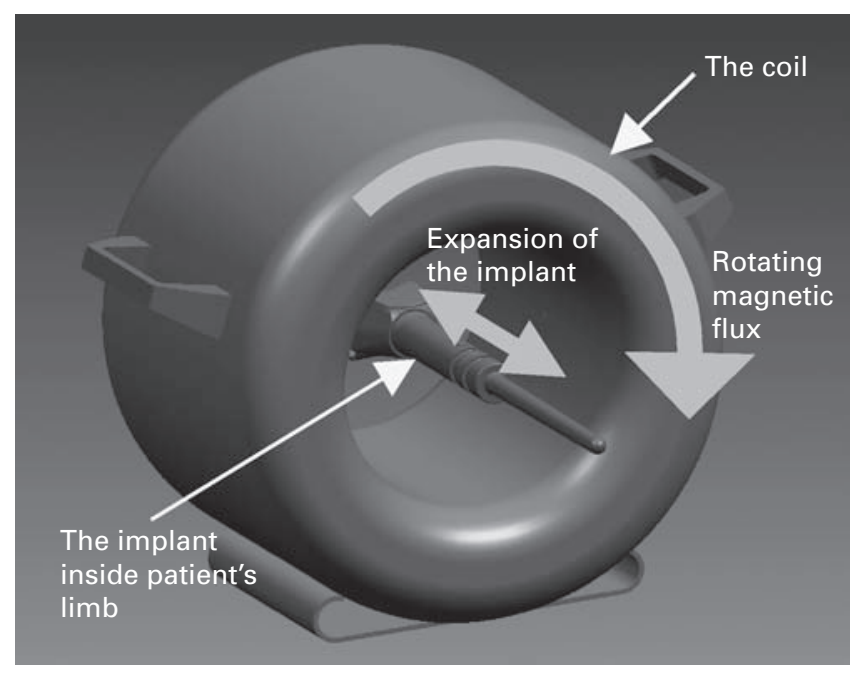

Fig. 2

A representation of the endoprosthesis in an external drive unit. As the external drive unit generates the electromagnetic field, it causes the magnetic disc in the prosthesis to rotate and to lengthen the prosthesis.

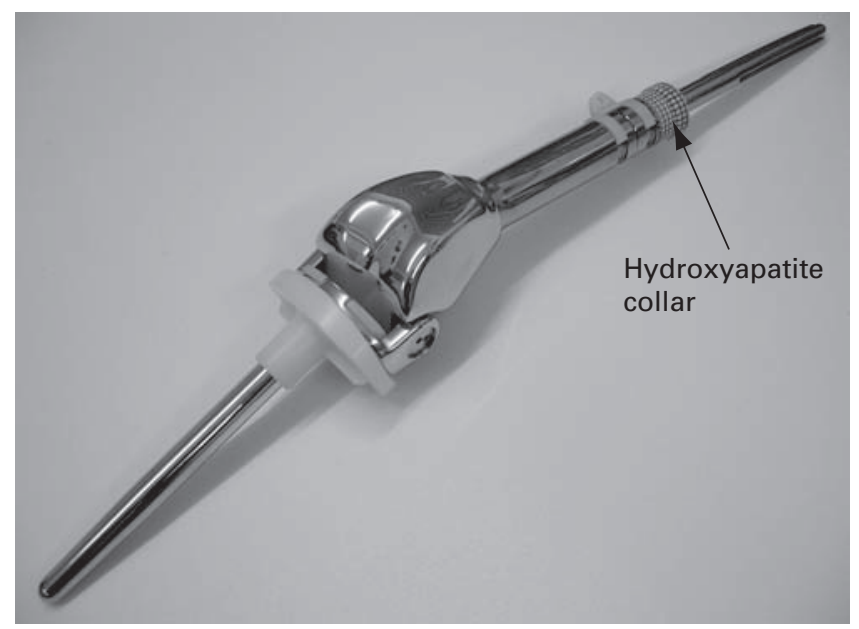

Fig. 3

The non-invasive distal femoral expandable endoprosthesis with a hydroxyapatite collar on the proximal femoral component.

The field strength between the magnet within the implant and the electromagnetic flux generates a coupling torque which is amplified through the gearbox to approximately 4 $\mathrm{Nm}$, in order to drive the power screw. This enables the prosthesis (Fig. 3) to withstand $1350 \mathrm{~N}$ of axial thrust during the lengthening process. The force generated is similar to that found during in vivo measurements, reported by Meswania et al, ${ }^{21}$ to overcome tension in the surrounding soft tissue, which increases as the implant is extended. Accordingly, to prevent the non-invasive implant being 


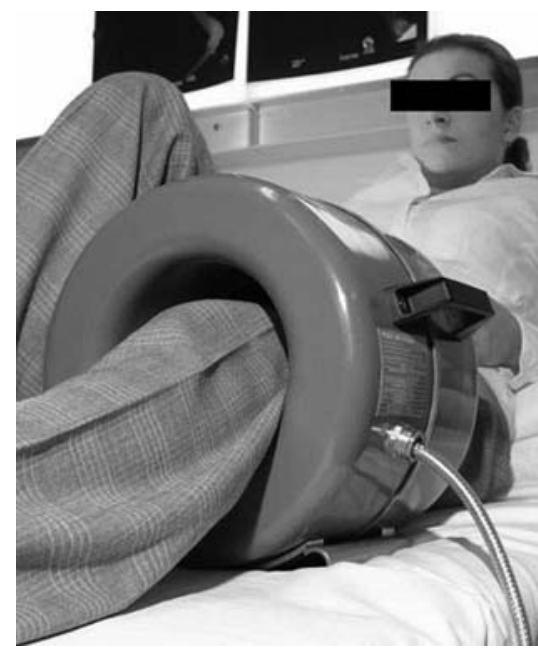

Fig. $4 a$

An 11-year-old patient, a) having a limb lengthened with the non-invasive distal femoral expandable endoprosthesis. The radiographs show the prosthesis, b) immediately post-operatively, and c) at the final follow-up of 30 months.

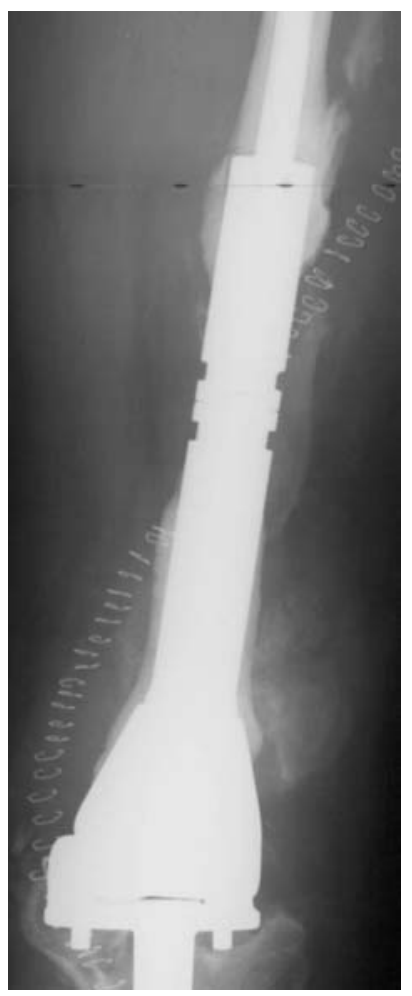

Fig. 4b

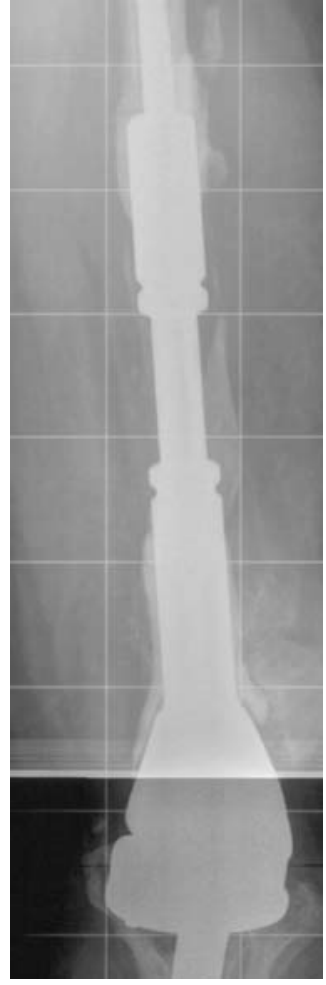

Fig. 4c

Table I. Details of non-invasive distal femoral expandable endoprosthesis in the limb salvage surgery of paediatric osteosarcomas

\begin{tabular}{|c|c|c|c|c|c|c|c|c|c|}
\hline $\begin{array}{l}\text { Patient } \\
\text { number }\end{array}$ & Gender & $\begin{array}{l}\text { Age } \\
\text { (yrs) }\end{array}$ & Reason for insertion & $\begin{array}{l}\text { Follow-up } \\
\text { (mths) }\end{array}$ & $\begin{array}{l}\text { Number of } \\
\text { lengthenings }\end{array}$ & $\begin{array}{l}\text { Amount of } \\
\text { lengthening } \\
(\mathrm{mm})\end{array}$ & $\begin{array}{l}\text { Range of } \\
\text { movement } \\
\left({ }^{\circ}\right)\end{array}$ & $\begin{array}{l}\text { MSTS }^{*} \\
\text { score }\end{array}$ & Outcome/complications $^{\dagger}$ \\
\hline 1 & $\mathrm{~F}$ & 9 & Osteosarcoma distal femur & 24 & 1 & 4.25 & 0 to 120 & 11 & Died of carcinomatosis \\
\hline 2 & $\mathrm{~F}$ & 10 & Osteosarcoma distal femur & 16 & 10 & 36 & 10 to 110 & 22 & \\
\hline 3 & $\mathrm{~F}$ & 11 & Osteosarcoma distal femur & 30 & 6 & 24 & 0 to 110 & 29 & \\
\hline 4 & $\mathrm{M}$ & 14 & Osteosarcoma distal femur & 14 & 6 & 20 & 5 to 100 & 20 & $25^{\circ}$ FFD-recovered \\
\hline 5 & M & 12 & Osteosarcoma distal femur & 20 & 7 & 28 & 5 to 120 & 21 & \\
\hline 6 & M & 14 & Osteosarcoma distal femur & 24 & 14 & 55 & 25 to 110 & 21 & $\begin{array}{l}\text { Needs revision to a new } \\
\text { NIG }\end{array}$ \\
\hline 7 & $\mathrm{~F}$ & 15 & $\begin{array}{l}\text { Revision of minimally invasive } \\
\text { expandable prosthesis }\end{array}$ & 14 & 2 & 8 & 0 to 110 & 20 & \\
\hline
\end{tabular}

* MSTS, Musculoskeletal Tumour Society

† FFD, fixed flexion deformity, NIG, non-invasive grower

overloaded, when it is extended it should be with small increments not exceeding $3 \mathrm{~mm}$ to $4 \mathrm{~mm}$.

The external drive unit is controlled by a power unit, which operates from a single phase 220 -volt power source. Both units are portable, with the coil weighing $32 \mathrm{~kg}$ and the power unit $16 \mathrm{~kg}$. This allows the external unit to be used in outpatient clinics. However, as the external coil has an internal diameter of $16 \mathrm{~cm}$ (50 cm circumference) this implant is not suitable for obese patients.

We have prospectively studied the early clinical results, in paediatric patients, of this prosthesis in limb-salvage oper- ations for osteosarcomas of the distal femur. We obtained local ethical committee approval to implant the prosthesis in seven patients (three boys and four girls), with a mean age of 12.1 years (9 to 15 ). The study was conducted between January 2002 and January 2004. There were four patients with a high grade, non-metastatic osteosarcoma of the distal femur who had received neoadjuvant chemotherapy. Metastatic disease was identified in two patients. One patient had the prosthesis implanted as a secondary procedure in order to replace a minimally invasive Stanmore Mark IV prosthesis, which had been maximally 
extended. The prosthesis was used in patients who had an existing or anticipated limb-length discrepancy of more than $2 \mathrm{~cm}$ and who were still growing. Each prosthesis was custom-designed, based upon pre-operative imaging, with consideration of the level of the intended femoral resection, predicted future growth and anticipated leg-length discrepancy. There was an understanding that in patients who received extensive chemotherapy, exact limb-length projections were difficult to forecast. ${ }^{22}$

The operations were performed by two of the senior authors (TWRB, SRC) at the time of primary tumour resection. Five prostheses were cemented into the intramedullary cavity of the remaining proximal femur and two were uncemented.

Post-operative chemotherapy was instituted when applicable, as were gentle extension-flexion exercises of the knee and weight-bearing when patients were able. The prosthesis could not be extended if the patient developed a fixed-flexion deformity, so maintaining full flexion was important.

The lengthenings were undertaken once a patient had developed at least $5 \mathrm{~mm}$ of limb-length discrepancy, except in the first case, when the lengthening was scheduled earlier to determine if the device would function when implanted. Generally the prosthesis was extended every four to six weeks in order to keep up with the growth of the contralateral limb, but sometimes more frequently in the event of a rapid growth spurt. Lengthening was only performed once the patient could flex the knee beyond $90^{\circ}$. Flexion stretched the soft tissues and reduced the load on the implant and gearbox during subsequent lengthenings.

Standard routine radiographs were obtained before lengthening, as well as after the first lengthening procedure, to determine whether the prosthesis had extended. Lengthening was performed at an outpatient clinic, without any anaesthesia. After each lengthening, a patient's neurovascular status was evaluated. Repeated applications of the external electromagnetic field were necessary in order to achieve the desired lengthening for several patients. At each attendance, the total lengthening was recorded for each patient, as well as the range of knee movement and any complications which occurred after extension. Patients were functionally evaluated at their last follow-up visit using the Musculoskeletal Tumour Society scoring system. ${ }^{23}$

\section{Results}

No patient reported pain during the lengthening process but as expansion occurred a stretching feeling was experienced. All patients were able to regain their usual level of function by the day after lengthening. One patient was unable to regain full extension after final lengthening, but after reversing the mechanism and shortening by $2 \mathrm{~mm}$ full extension was regained. There was no heating of the implant during lengthening. The noise of the gearbox, when energised, could be detected using a stethoscope placed on the greater trochanter. The number of lengthen- ing procedures for each patient varied from one to 14 . All the patients were able to walk unaided after the procedure. The mean time from the implantation to the last follow-up was 20.2 months (14 to 30 ). The mean lengthening achieved during each procedure was $4 \mathrm{~mm}$ and no neurovascular compromise occurred. Patients were lengthened by a mean of $25 \mathrm{~mm}$ (4.25 to 55) (Fig. 4), the mean knee flexion angle being $110^{\circ}$ (Table I).

There has been no failure of lengthening, although one patient required simple traction to his ankle at the time of expansion to aid the process. The resistance to lengthening in this case was attributed to well-developed muscles around the thigh. Six patients are still undergoing active expansion. One patient has achieved $55 \mathrm{~mm}$ of lengthening in the prosthesis, while the maximum length of extension which can be achieved in the design is $60 \mathrm{~mm}$. As a result, this patient will require revision surgery in order to replace the existing implant with another non-invasive expandable endoprosthesis to accommodate further lengthening as is necessary while growth continues.

The mean Musculoskeletal Tumour Society score was 20 of 30 (11 to 29 ). There were only two complications during the study. One patient developed a flexion deformity at the knee of $25^{\circ}$ at four months and one died from disseminated disease. The former required manipulation under anaesthesia and serial casting.

There have been no cases of stem fracture, implant failure, aseptic loosening, infection or local tumour recurrence. For post-operative management and treatment, these patients were able to receive radiographic and CT assessments, but were unable to have MRI because of magnetic interference.

\section{Discussion}

The treatment of malignant bone tumours in skeletally immature patients has long been a challenge to orthopaedic oncologists. Limb-preservation surgery for patients with bone tumours is an established treatment, but in skeletal immaturity limb-length inequality at the end of the growth phase is likely. Endoprostheses have been developed to address this issue. Earlier designs were modular, in which mid-sections were exchanged for progressively longer sections. These were superseded by minimally invasive designs, which required a small incision under anaesthesia in order to expand the prosthesis, although this risked anaesthetic complications and infection.

The first designs capable of non-invasive expansion were introduced in the 1990s. ${ }^{17-20}$ The Phenix prosthesis (Phenix Medical, Paris, France $)^{18}$ became available in 1990 . The lengthening in this prosthesis depended on the controlled melting of a polyethylene tube with a spring, using an external electromagnetic field. As the polyethylene tube melted, it allowed controlled release of the spring's energy so that the prosthesis could extend. In practice, $6 \mathrm{~mm}$ to 15 $\mathrm{mm}$ of lengthening was achievable at each expansion, depending on the patient, the state of soft tissues, the residual force of the spring and the location of the prosthesis. 
Wilkins and Soubeiran ${ }^{18}$ published their early experience with the Phenix expandable prosthesis. They inserted seven prostheses in six patients. Of the seven implants, six were used during revision procedures in salvage situations and only one was implanted during an index procedure. One patient sustained a fracture of the prosthesis in a fall and developed an infection after implantation of a second prosthesis. There were 21 expansions performed in six patients (mean lengthening at each procedure, $8 \mathrm{~mm}$ ). Prosthetic expansions took a mean of 20 to 30 seconds and were accompanied by very mild discomfort, if any. An oral analgesic was administered to most patients either before or during the lengthening procedure.

Neel et $\mathrm{al}^{20}$ reported a multicentre experience with 18 Phenix non-invasive expandable prostheses in 15 patients with osteosarcoma of the distal femur. They conducted 60 expansions in these patients. Of the 15 original prostheses, ten were implanted at the time of primary tumour resection and five were revisions from an endoprosthetic modular knee system. Of the 60 expansions, all but two were performed as outpatient procedures. A mean of $8.5 \mathrm{~mm}$ was obtained at each lengthening (1 to 30). The mean follow-up was 21.5 months (12 to 33 ) and the final mean Musculoskeletal Tumour Society functional score was $90 \%$. Eight revisions were required for stem fracture or loosening. There was one amputation because of a post-operative arterial thrombosis.

Our study reports the initial experience with the noninvasive distal femoral expandable endoprosthesis in seven patients. Only one patient developed a flexion deformity at the knee of $25^{\circ}$, which we believe was caused by repeated lengthenings at shorter intervals of time, without letting the soft tissues stretch adequately. The deformity was corrected with the use of serial plasters. We also noted that immediately after the lengthening, the patients had a reduced range of knee movement on the day of lengthening. Increasing tension in the muscle envelope in the thigh during the lengthening process may be the cause of this. However, exercise after lengthening restored the range of movement to normal. We feel that early and regular physiotherapy is important to maintain a good range of movement, prevent scar formation and allow subsequent extension of the prosthesis to be achieved with less force on the gearing mechanism.

In our series there has been no prosthetic failure or stem fracture and no evidence of loosening. This latter finding could be because of the use of a hydroxyapatite-coated ${ }^{24}$ collar on the proximal stem of the femoral component, permitting better bone ingrowth and reducing the chance of aseptic loosening.

This prosthesis may not be appropriate for very young patients, where the length of bone resection is short. Currently there are three length options; 50, 70 and $90 \mathrm{~mm}$ growth sections. The minimum possible length of resection which can accommodate a complete prosthesis is $170 \mathrm{~mm}$ in the distal femur, measured from the joint line. If a longer resection is required, the prosthesis is customised to match the resection.

In comparison with other non-invasive devices, our noninvasive expandable endoprosthesis differs in two main aspects. First, the prosthesis stays in situ indefinitely and does not need to be changed at the end of the growth period. Revision is only likely to be necessary if the patient grows in excess of the maximum possible extension of the implant. Secondly, the lengthenings are achieved in measureable and controlled amounts of $0.23 \mathrm{~mm}$ per minute.

This design is still under development but the authors are optimistic that it will obviate some of the problems encountered in limb preservation in the growing child. The prosthesis allows periodic, small, measurable and predictable extensions, which are physiologically more favourable than major surgical interventions which stretch the soft tissue of the limb to a maximum degree. The prosthesis also avoids multiple surgical procedures and general anaesthesia, while offering lower morbidity, cost savings and less psychological trauma. This unique prosthesis has shown promising early results, but additional data are required about its long-term structural integrity.

Special thanks to Chris Henry and Rosemary Dutton of the Bone Tumour Unit at the Royal National Orthopaedic Hospital. The authors also acknowledge the contribution made to the project by Stanmore Implants Worldwide.

No benefits in any form have been received or will be received from a commercial party related directly or indirectly to the subject of this article.

\section{References}

1. O'Flanagan SJ, Stack JP, McGee HM, Dervan P, Hurson B. Imaging of intramedullary tumour spread in osteosarcoma: a comparison of techniques. J Bone Joint Surg [Br] 1991;73-B:998-1001.

2. Lewis MM. An approach to the treatment of malignant bone tumors. Orthopedics 1985;8:655-6.

3. Link MP, Goorin AM, Miser AW, et al. The effect of adjuvant chemotherapy on relapse-free survival in patients with osteosarcoma of the extremity. $N$ Engl $\mathrm{J}$ Med 1986;314:1600-6.

4. Rosen G, Murphy ML, Huvos AG, Gutierrez M, Marcove RC. Chemotherapy, en bloc resection, and prosthetic bone replacement in the treatment of osteogenic sarcoma. Cancer 1976;37:1-11.

5. Simon MA, Aschliman MA, Thomas N, Mankin HJ. Limb-salvage treatment versus amputation for osteosarcoma of the distal end of the femur. J Bone Joint Surg [Am] 1986;68-A:1331-7.

6. Rougraff BT, Simon MA, KneisI JS, Greenberg DM, Mankin HJ. Limb salvage compared with amputation for osteosarcoma of the distal end of the femur: a longterm oncological, functional, and quality-of-life study. J Bone Joint Surg [Am] 1994; 76-A:649-56.

7. Springfield DS, Schmidt R, Graham-Pole J, et al. Surgical treatment for osteosarcoma. J Bone Joint Surg [Am] 1988;70-A:1124-30.

8. Eckardt JJ, Eilber FR, Dorey FJ, Mirra JM. The UCLA experience in limb salvage surgery for malignant tumors. Orthopedics 1985;8:612-21.

9. McDonald DJ. Current concepts of treatment of bone tumors. In: Schajovicz F, ed Tumors and tumor like lesions of bone: pathology, radiology and treatment. New York: Springer-Verlag, 1994:613-43

10. Eckardt JJ, Kabo JM, Kelley CM, et al. Expandable endoprosthesis reconstruction in skeletally immature patients with tumors. Clin Orthop 2000;373:51-61.

11. Lewis MM. The use of an expandable and adjustable prosthesis in the treatment of childhood malignant bone tumors of the extremity. Cancer 1986;57:499-502.

12. Lewis MM, Bloom N, Esquieres EM, Kenan S, Ryniker DM. The expandable prosthesis: an alternative to amputation for children with malignant bone tumors. AORN J 1987;46:457-70.

13. Scales JT, Sneath RS. The extending prosthesis. In: Coombs R, Friedlaender G, eds Bone tumour management. London: Butterworth \& Co., 1987:168-77.

14. Schindler OS, Cannon SR, Briggs TW, Blunn GW. Stanmore custom-made extendible distal femoral replacements: clinical experience in children with primary malignant bone tumours. J Bone Joint Surg [Br] 1997;79-B:927-37. 
15. Eckardt JJ, Safran MR, Eilber FR, Rosen G, Kabo JM. Expandable endoprosthetic reconstruction of the skeletally immature after malignant bone tumor resection. Clin Orthop 1993;297:188-202.

16. Schindler OS, Cannon SR, Briggs TW, et al. Use of extendable total femoral replacements in children with malignant bone tumors. Clin Orthop 1998;357: 157-70.

17. Baumgart R, Betz A, Schweiberer L. A fully implantable motorized intramedullary nail for limb lengthening and bone transport. Clin Orthop 1997;343:135-43.

18. Wilkins RM, Soubeiran A. The Phenix expandable prosthesis: early american experience. Clin Orthop 2001;382:51-8.

19. Gitelis S, Neel MD, Wilkins RM, et al. The use of a closed expandable prosthesis for pediatric sarcomas. Chir Organi Mov 2003;88:327-33.
20. Neel MD, Wilkins RM, Rao BN, Kelly CM. Early multicenter experience with a noninvasive expandable prosthesis. Clin Orthop 2003;415:72-81.

21. Meswania JM, Walker PS, Sneath RS, Grimer RJ. In vivo distraction forces in extendible endoprosthetic replacements: a study of 34 patients. Proc Inst Mech Eng [H] 1988;212:151-5.

22. Cool P, Davies M, Grimer RJ, Carter SR, Tillman RM. Growth in the lower limb following chemotherapy for a malignant primary bone tumour: a straight-line graph. Sarcoma 1997;1:75-7.

23. Enneking WF, Dunham W, Gebhardt MC, Malawer M, Pritchard D. A system for the functional evaluation of reconstructive procedures after surgical treatment of tumors of the musculoskeletal system. Clin Orthop 1993;286:241-6.

24. Geesink RG, de Groot K, Klein CP. Bonding of bone to apatite coated implants. J Bone Joint Surg [Br] 1988;70-B:17-22. 